\title{
Spirometry in primary care: An analysis of the first 100 patients referred in one general practice
}

\author{
H Pinnock, J Carley-Smith and D Kalideen
}

\begin{abstract}
SUMMARY
Aims: To assess the outcome of spirometry in primary care.

Methods: Analysis of the first 100 spirometry results undertaken in one general practice.

Results: Chronic obstructive pulmonary disease was diagnosed in 50 patients, asthma in 13 and a restrictive ventilatory disorder in $12 ; 2$ patients had mixed disease, 23 patients had normal spirometry. Conclusion: The results of spirometry considered with the clinical history allowed a diagnosis to be clarified in the majority of patients.
\end{abstract}

\section{INTRODUCTION}

The publication of the BTS guidelines for the management of chronic obstructive pulmonary disease (COPD) ${ }^{1}$ has stimulated interest in lung function testing within primary care. Of 582 randomly selected practices that were sent a questionnaire in 1997, 186 had a spirometer ${ }^{2}$ and a telephone survey in 1998 that produced similar results. ${ }^{3}$ In the majority of practices, the nurse was responsible for performing the measurements. This report considers the practical application of providing a spirometry service in general practice.

\section{METHODS}

In 1996, we began to offer lung function testing at the Whitstable Health Centre, using a Microlab 3,300 spirometer (Micromedical Ltd, Rochester, Kent) which provides a printout including a volume-time curve and a flow-volume loop. The manufacturer's instructions state that recalibration is not required between annual services. The tests were undertaken during a weekly clinic by a respiratory nurse whose COPD training had included basic spirometry.

A postgraduate education allowance (PGEA; part of the continuing medical education system in the UK) -approved lunchtime meeting was held in the practice for all partners and respiratory nurses to discuss the role of spirometry and the use of reversibility tests in the diagnosis of COPD. GPs referred patients for spirometry by completing a form which asked for background information about the patient, including an anticipated diagnosis.

Referring doctors indicated whether they wished the nurse to proceed to bronchodilator and steroidreversibility testing according to the practice protocol drawn up in accordance with the BTS guidelines (see Box). Completed tests were reviewed by one of the partners (HJP), and a conclusion was reached after consideration of the available clinical history, results of the spirometry and, where appropriate, a discussion with the referring doctor. The results of the first 100 patients referred for this service are analysed here.

\section{RESULTS}

The age range of patients referred for spirometry was $28-84$ years (mean age 65 years; $44 \%$ male), although the majority $(60 \%)$ of the patients were aged between 60-80 years; $26 \%$ were active smokers, $54 \%$ were exsmokers and $20 \%$ had never smoked. Of these patients, $23 \%$ had only baseline spirometry, $77 \%$ had bronchodilator reversibility and $41 \%$ proceeded to a steroid challenge.

As a result of baseline spirometry, $65 \%$ were classified with an obstructive ventilatory disorder $\left(\mathrm{FEV}_{1}<80 \%\right.$ and $\left.\mathrm{FEV}_{1} \%<70 \%\right), 12 \%$ had a restrictive ventilatory disorder and $23 \%$ had normal spirometry. Of the 65 patients with obstruction, 63 proceeded to reversibility tests; the results are shown in Table 1. Two patients declined bronchodilator reversibility and 11 did not proceed to an oral steroid challenge (nine had contraindications or their GP indicated that steroids were not to be used and four declined or did not complete the course). A comparison of anticipated diagnoses with the final conclusions is shown in Table 2.

\section{DISCUSSION}

The original intention on offering spirometry at Whitstable Health Centre was to differentiate between asthma and COPD, and to assess the severity and steroid responsiveness of those patients with COPD.

One of the unexpected outcomes was the identification of 12 patients with a restrictive ventilatory disorder. The restrictive nature of the problem had not been anticipated by the referring GP and, in seven cases, the anticipated diagnosis was COPD. The causes of the restriction were varied and included a past history of tuberculosis, scoliosis, a neurological deficit and one probable fibrosing alveolitis. Appreciating that the patients' dyspnoea was due to restrictive lung disease allowed

Practice protocol for reversibility testing

- Baseline

Patients should be stable at the time of the test (for example, at least six weeks since an exacerbation) and not have had a bronchodilator prior to testing (for example, no short-acting bronchodilator for six hours, no long-acting bronchodilator for 12 hours and no theophylline for 24 hours)

- Bronchodilator reversibility if $\mathrm{FEV}_{1}<80 \%$ and $\mathrm{FEV}_{1} \%<70 \%$ using salbutamol $5 \mathrm{mg}$ via a nebuliser

- Steroid reversibility if $\mathrm{FEV}_{1}<60 \%$ and $\mathrm{FEV}_{1} \%$ $<70 \%$ using prednisolone $30 \mathrm{mg}$ daily for two weeks
Hilary Pinnock

General Practitioner

Jane Carley-Smith

Practice Nurse

Doreen Kalideen

Practice Nurse

Whitstable Health Centre, Harbour Street, Whitstable, Kent CT5 1BZ, UK

Correspondence to: Dr H Pinnock

hpinnock@gpiag-asthma.org

Date received: 01/02/99

Date accepted: 01/04/99

Asthma Gen Pract 1999; 7(2): $23-4$ 
Table 1 Outcome of reversibility testing ( $n=65$ patients)

\begin{tabular}{|c|c|c|c|}
\hline & Obstructive (n) & Final diagnosis ( $n$ ) & $\begin{array}{l}\text { Prescribed } \\
\text { inhaled } \\
\text { steroids }(n)\end{array}$ \\
\hline $\begin{array}{l}\text { Baseline } \\
\text { testing }\end{array}$ & $\begin{array}{l}65 \\
\text { (15 mild, } 30 \text { moderate, } \\
20 \text { severe) }\end{array}$ & & 34 \\
\hline $\begin{array}{l}\text { Bronchodilator } \\
\text { reversibility } \\
n=63\end{array}$ & $\begin{array}{l}44 \text { No reversibility } \\
19 \text { Significant reversibility a }\end{array}$ & $\begin{array}{l}35 \text { COPD } \\
6 \text { Asthma fixed } \\
3 \text { Mixed } \\
12 \text { COPD }^{b} \\
4 \text { Asthma fixed } \\
3 \text { Asthma }^{\circ}\end{array}$ & \\
\hline $\begin{array}{l}\text { Steroid } \\
\text { reversibility } \\
n=39\end{array}$ & $\begin{array}{l}33 \text { No reversibility } \\
6 \text { Significant reversibility }\end{array}$ & $\begin{array}{l}27 \text { COPD } \\
4 \text { Asthma } \\
2 \text { COPD } \\
2 \text { Mixed } \\
2 \text { Asthma fixed }\end{array}$ & $\begin{array}{r}12 \\
3 \\
1 \\
2 \\
1\end{array}$ \\
\hline
\end{tabular}

a. Increase in $\mathrm{FEV}_{1}$ that is both $>200 \mathrm{ml}$ and $15 \%$ over the prebronchodilator value ${ }^{1}$

b. Reversibility which fell between $200-500 \mathrm{ml}$ was interpreted after consideration of the clinical history and the shape of the flow volume loop

c. A substantial response in $\mathrm{FEV}_{1}(>500 \mathrm{ml})$ was taken to indicate asthma

Table 2 Comparison of anticipated diagnosis with final conclusion

\begin{tabular}{l|ccccc}
\multirow{2}{*}{$\begin{array}{l}\text { ANTICIPATED } \\
\text { DIAGNOSIS }\end{array}$} & Asthma & COPD & \multicolumn{6}{c}{ DIAGNOSIS } \\
Mixed & Restrictive & Normal \\
\hline Asthma & 6 & 2 & 0 & 1 & 10 \\
\hline COPD & 3 & 36 & 1 & 7 & 8 \\
\hline Mixed & 4 & 7 & 1 & 3 & 2 \\
\hline Other/not stated & 0 & 5 & 0 & 1 & 3
\end{tabular}

appropriate management - or possibly prevented inappropriate treatment.

The spirometry results were interpreted together with the clinical history provided by the referring doctor and medical records which included smoking history, age at onset of symptoms and previous peak expiratory flow readings. This allowed differentiation of COPD from asthma in the majority of the 65 patients with obstructive lesions. In three cases, a clear diagnosis could not be made. Six patients had a definite history of asthma and their fixed obstruction was considered to be due to remodelling (changes occurring in the airways of asthmatics which result in irreversibility) rather than smoking. Three patients had an increase in $\mathrm{FEV}_{1}$ of more than $500 \mathrm{ml}$ and a diagnosis of asthma was confirmed in accordance with the BTS guidelines. ${ }^{4}$

Steroid reversibility was carried out in 39 of the 65 patients with obstruction. Of the 29 with COPD, 27 did not have steroid reversibility. Twelve were already taking inhaled steroids and the intention was to withdraw the inhaled steroids, cautiously, because of the concern that current use of inhaled steroids may have masked potential reversibility.

Spirometry proved to be relatively unhelpful in the diagnosis of asthma; 10 of the 19 patients referred as probable asthmatics had normal lung function. Asthma is a dynamic condition and normal spirometry at one point in time does not exclude the diagnosis. Peak expiratory flow diary charts are more appropriate for confirming asthmatic variability.

Technical problems were detected in $73(33 \%)$ of the 218 sets of analysed readings (100 baseline, 77 bronchodilator reversibility and 41 steroid trials). In 51 of these sets, there was more than $5 \%$ variability, which falls short of the standards set by the BTS. However, there are practical reasons why the standards cannot always be achieved. Some patients have considerable difficulty performing an adequate blow and it may be neither humane nor productive to repeat blows in the hope of obtaining two perfect readings. Age appeared to be a major factor influencing the reproducibility of the tests. For readings with less than $5 \%$ variability, the average age was 54 years whereas for readings with more than $5 \%$ variability, the mean age was 71 years. The guidelines recognise that spirometry may be exhausting and recommend a maximum of six blows. When this limit was reached or the patient was clearly unable to undertake another blow, the test was halted and the best reading selected. Graphs were useful in deciding the quality of manoeuvres, and we adopted a policy of always printing the results of all blows so that the interpretation could take this into account.

An incomplete blow was the next most common fault and was noted in 22 readings. A possible explanation for these technical problems is that the spirometer used displayed a flow-volume loop which does not allow for easy assessment of the completion of the blow. More recent models display a volumetime graph which may help to overcome these problems as it is comparatively easy to identify when a plateau has been reached.

Spirometry has proved to be a practical proposition in this primary care setting. The results of baseline and reversibility tests, taken in conjunction with the clinical history, allowed a diagnosis to be clarified in the majority of patients.

\section{References}

1. BTS guidelines for the management of chronic obstructive pulmonary disease. Thorax 1997;52(5):S1-28

2. Bellamy D, Hoskins G, Smith B, et al. The use of spirometers in general practice. Asthma Gen Pract 1997;5(1):8-9

3. Dowson LJ, Yeung A, Allen MB. Most practices would use open access spirometry in hospitals. BMJ 1998;317:209

4. British Thoracic Society COPD guidelines summary. Thorax 1997;52(5):S1-32 